\title{
Los estudios sobre el riñón y la circulación renal de Josep Trueta i Raspall (1897-1977)
}

\author{
Fariña Pérez LA.
}

\author{
Urología, Hospital Povisa. Vigo (Pontevedra).
}

Actas Urol Esp. 2008;32(3):276-280

\section{RESUMEN}

LOS ESTUDIOS SOBRE EL RIÑÓN Y LA CIRCULACIÓN RENAL DE JOSEP TRUETA i RASPALL (1897-1977)

Introducción: Josep Trueta i Raspall, nacido en Barcelona en 1897, fue uno de los más destacados cirujanos de su tiempo. Después de iniciada la rebelión militar y la guerra de 1936, se hace cargo de la cirugía en el Hospital de la Santa Creu y Sant Pau y pone en práctica un método de cura oclusiva de las fracturas abiertas, que disminuye dramáticamente el número de gangrenas y amputaciones entre civiles y militares. Con el final de la guerra y el exilio, es invitado por el servicio de salud inglés, en Oxford enseña sus conocimientos de cirugía de guerra, es nombrado doctor honoris causa y profesor de ortopedia, y crea una escuela de investigación clínica que genera importantes hallazgos en el desarrollo y patología del hueso y, entre otras áreas, en el conocimiento de la función renal y las lesiones renales inducidas por el shock y la hipertensión arterial. Uno de los frutos más destacados de esta investigación es el libro "Estudios sobre la circulación renal", publicado en inglés en 1947 (Oxford) y en castellano en 1949 (Barcelona).

Material y Método: Estudio de la biografía de Josep Trueta y análisis de sus trabajos sobre la función renal, en especial del texto "Estudios sobre la circulación renal", relacionándolo con el estado de los estudios nefrológicos en su tiempo.

Resultados: Trueta y su grupo fueron pioneros en el estudio de las causas de la oliguria postraumática de los heridos de guerra. Basándose en los primeros estudios sobre el sindrome de aplastamiento, publicados en 1941, en los que se señalaba el fallo renal que seguía a las lesiones extensas de los miembros, demuestran los cambios en la circulación renal consecutivos a diferentes lesiones de los miembros, como isquemia, hemorragia e infecciones. "Estudios sobre la circulación renal" y otros textos publicados en revistas de gran impacto, exponen la isquemia cortical renal con preservación o aumento de la circulación medular y yuxtamedular que tiene lugar en estos estados patológicos. Aunque sus aportaciones y las hipótesis que aventuró, no fueron definitivas, sirvieron de base para otros estudios realizados años después sobre la insuficiencia renal aguda secundaria al shock, la glomerulonefritis, el rechazo del trasplante y la hipertensión arterial.

Comentario: Los trabajos de Trueta son un extraordinario ejemplo de investigaciones dirigidas a responder a preguntas clínicas concretas. Llama la atención el amplio campo de investigación en que se mueve, que hoy día implicaría a diversas especialidades muy ajenas a sus tareas de cirujano ortopeda. Además de su pasión catalanista, que le movió a escribir "The spirit of Catalonia" (1946), un breve texto sobre la historia de Cataluña para el público de habla inglesa, Trueta fue un intelectual humanista con intereses muy amplios, que dejó reflejados en sus libros de viajes, y en textos sobre la vida de sabios clásicos como Luis Vives, Miguel Servet o Cajal. Regresó a Cataluña en 1966 y falleció en 1977. Su libro "Estudios sobre la circulación renal", exquisitamente ilustrado y editado, merece un lugar destacado en la historiografía médica de interés urológico.

Palabras clave: Historia de la urología. Historia de la nefrología.

\section{ABSTRACT}

STUDIES ON THE KIDNEY AND THE RENAL CIRCULATION, BY JOSEP TRUETA I RASPALL (1897-1977)

Introduction: Josep Trueta i Raspall, born in Barcelona in 1897, was one of the most outstanding european surgeons of his time. In 1936, after the military coup and during the subsequent Spanish civil war, he was in charge of the Surgery Service at the Hospital de la Santa Creu y Sant Pau and begins to performe a closed method to treat open fractures of the limbs, dramatically lowering the number of gangrenes and amputations between troops and civilians. At the end of the war came the exile, and he was invited by the british Health Service, he teach in Oxford and applies his knowledge on war surgery, was named $\mathrm{PhD}$ (Hon) and professor of ortopedics, and creates an school of clinical investigation that generates important findings in bone growing and pathology and, amont other areas, in the knowledge of renal function and kidney injuries induced by shock and blood hypertension. One of the most outstanding fruits of this investigation is the book "Studies on renal circulation", first published in English in 1947 (Oxford), then in Spanish in 1949 (Barcelona).

Material and Method: Study of Josep Trueta's biography and works on kidney function, especially the book "Studies on the renal circulation", relating them to the state-of- the- art of nephrologic studies of his time.

Results: Trueta and his group were pioneers in the study of the causes of postraumatic oliguria in war wounds. On the basis of the first studies on crush syndrome, published in 1941, were the kidney failure that followed the extended injuries of the limbs was pointed out, they could demonstrated the changes in renal circulation that followed different injuries to the limbs, such as ischemia, haemorrhage and infections. "Studies on the renal circulation" and other papers published in journals of high scientific impact, shown the renal cortical ischemia with preservation or increase of the medullar and yuxtamedullar circulation that takes place in those pathological situations (Trueta shunt). Although their contributions and hypothesis were not definitives, they served as the basis to studies performed later on, on acute kidney failure secondary to shock, glomerulonephritis, transplant rejection and blood hypertension.

Comment: Trueta's works are unusual examples of investigations directed to answer clinical questions. What may calls one's attention is the wide field of investigation he moves on, that today would involved several medical specialities, different to his task as orthopedic surgeon. On the other hand, his love by Catalonia, his country, moves him to write "The spirit of Catalonia" (1946), a short text for English-speaking people, on the political and scientific history of Catalonia. Trueta was in the end, an humanistic intellectual with broad interests, reflected on his books on travels or on the life of classic sages such as Luis Vives, Miguel Servet or Cajal. He returned back to Catalonia in 1966 and died in 1977 . His book "Studies on the renal circulation", exquisitely illustrated and edited deserves a distinguished place in the European medical and urological historiography.

Keywords: History of urology. History of nephrology. 
$\mathrm{J}$

osep Trueta i Raspall, nacido en Barcelona en

1897 y graduado en esa Facultad de Medicina en 1921, fue uno de los más destacados cirujanos europeos de su tiempo. Desde 1921 trabaja en el Hospital de Santa Creu de esa ciudad (más tarde en el nuevo Hospital de Santa Creu y San Pau), como ayudante del Dr Manuel Corachán, y después de la rebelión militar fascista que dio lugar al inicio de la Guerra Civil Española de 1936, se hace cargo del Servicio de Cirugía A y pone en práctica un método novedoso de cura oclusiva de las fracturas abiertas. Estaba basado en el método del cirujano norteamericano Orr de inmovilización para las osteomielitis crónicas, pero asegurando la limpieza y escisión de los tejidos enfermos en las heridas y fracturas recientes. Consistía por tanto en el tratamiento quirúrgico inmediato, mediante limpieza de la herida, escisión de esfacelos, drenaje e inmovilización con vendaje de yeso, y disminuyó dramáticamente el número de gangrenas y amputaciones entre civiles y militares con heridas de guerra.

En 1939, con el final de la Guerra Civil, elige junto a su familia el exilio, y es invitado por el Servicio de Salud inglés, deseoso de contar con su experiencia para el conflicto que se esperaba en Europa. Trabaja en el Nuffield Orthopaedic Hospital de Oxford (patrocinado por el fabricante de automóviles Wingfield Morris, lord Nuffield), es nombrado doctor honoris causa (Universidad de Oxford, 1942) y profesor de ortopedia (Figs. 1 y 2), y enseña y aplica sus conocimientos de cirugía de guerra, que fueron ampliamente usados por muchos de los ejércitos que lucharon en la Segunda Guerra Mundial y también en las guerras de Corea y Vietnam. Su libro "The principles and practice of war surgery: with reference to the biological method of the treatment of war wounds and fractures" (London, 1943), fue un texto definitivo en esos años, así como "An atlas of traumatic surgery. Illustrated histories of wounds of the extremities" (Oxford, 1949).

También en Oxford participa en los estudios clínicos iniciales con la penicilina, el primer antibiótico, llevados a cabo por el grupo dirigido por Howard W. Florey y Ernst B.Chain (Fig. 3), y que les valió el premio Nóbel de Fisiología y Medicina en el año 1945 a éstos y a Alexander Fleming, quien descubriera la sustancia en el año 1928. En esa ciudad inglesa Trueta creó una escuela de investigación clínica que generó importantes investigaciones en el

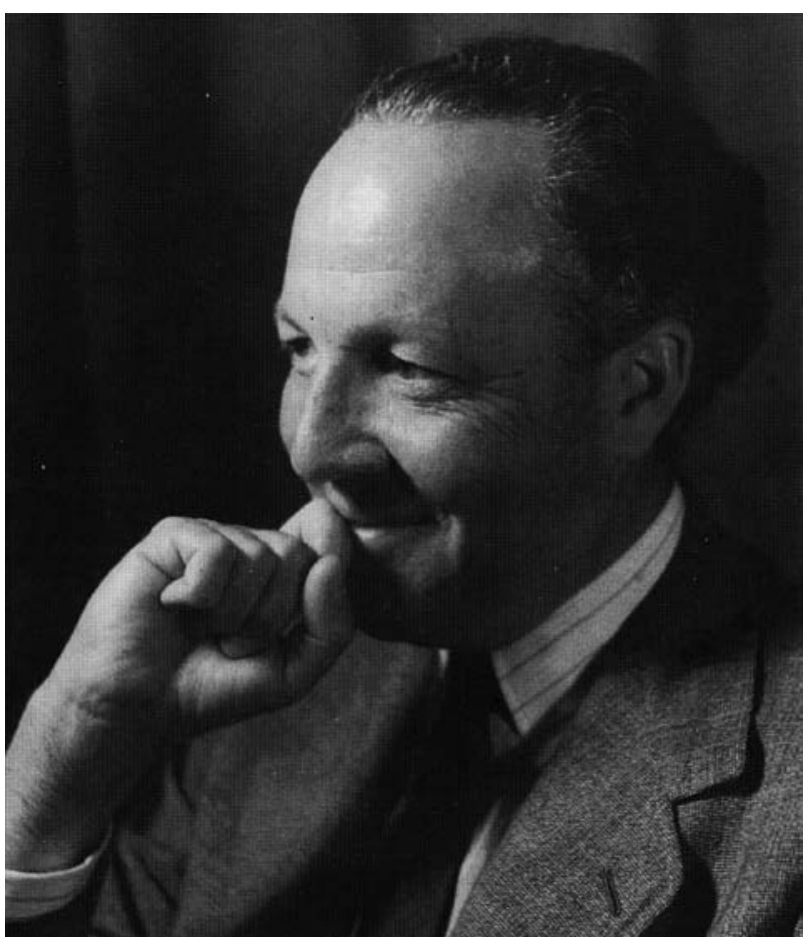

FIGURA 1. Josep Trueta en Oxford (1944).

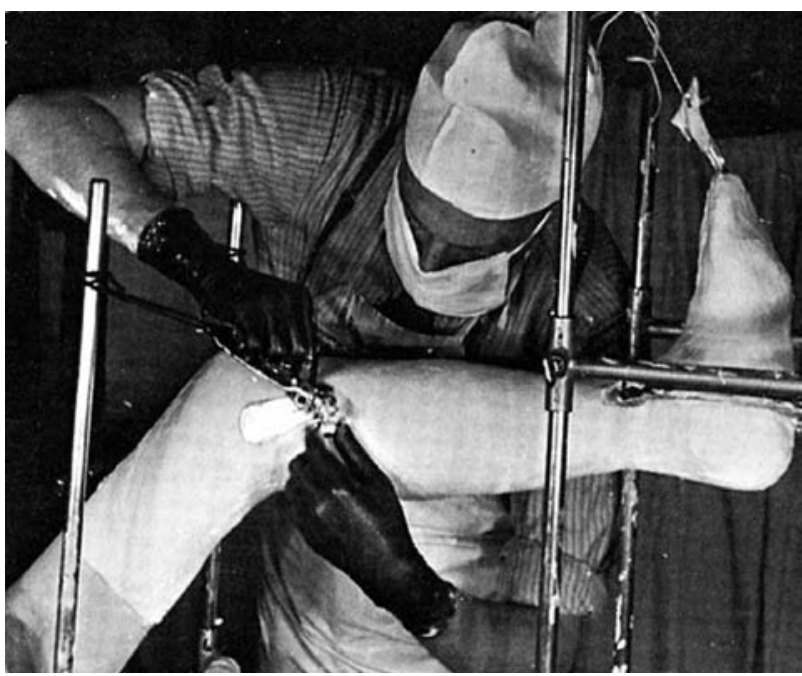

FIGURA 2. Trueta realizando una de sus "curas oclusivas" de traumatismo abierto de la extremidad inferior.

desarrollo y patología del hueso, algunas de las cuales siguen vigentes, y, entre otras áreas, en el conocimiento de la función renal y las lesiones renales inducidas por el shock y la hipertensión arterial ${ }^{1-6}$.

Uno de los frutos más destacados de estas últimas investigaciones es el libro "Estudios sobre la circulación renal”, publicado en inglés en 1947 y en

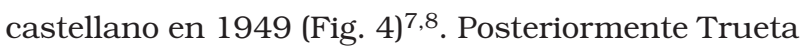
concentró sus investigaciones en el estudio de la 


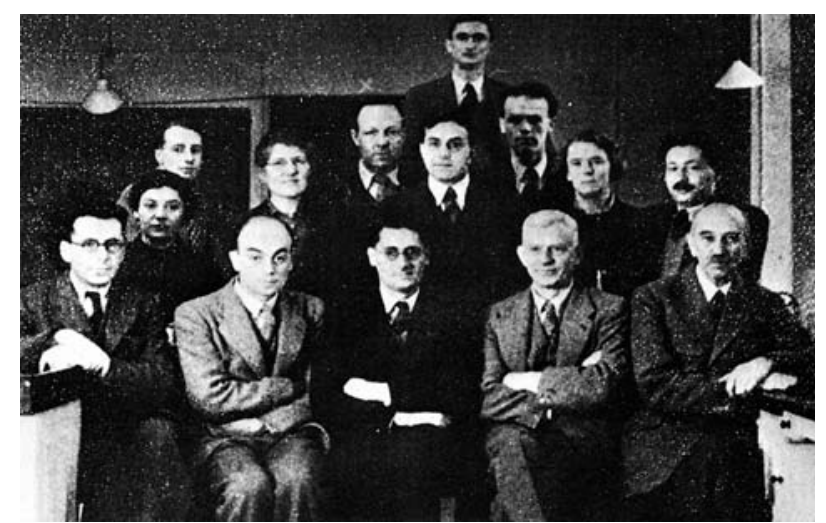

FIGURA 3. Trueta (X) con el grupo de investigadores de la penicilina en Oxford (1941). En la primera fila los premios Nobel Howard W. Florey (centro) y Ernst B. Chain (a su derecha).

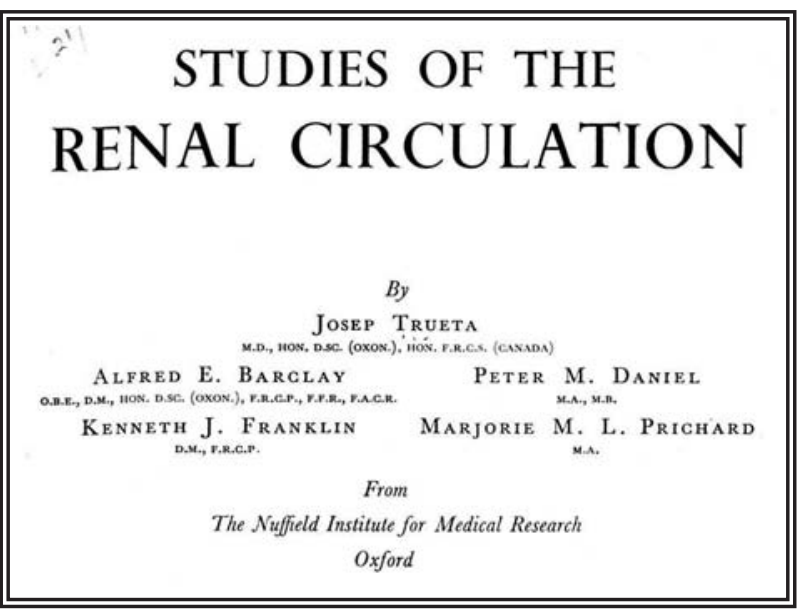

FIGURA 4. Página de portada de la edición inglesa de "Studies on the renal circulation" (Oxford 1947).

osteogénesis y el crecimiento del hueso. Su bibliografía incluye 11 libros y 149 artículos, la mayor parte publicados en revistas que en esos años tenían gran impacto científico.

\section{MATERIAL Y MÉTODO}

Estudio de la biografía de Josep Trueta y análisis de sus trabajos sobre la función renal, en especial del texto "Estudios sobre la circulación renal", relacionándolo con el estado de los estudios nefrológicos en su tiempo.

\section{RESULTADOS}

Trueta fue un pionero en el estudio de las causas de la oliguria postraumática de los heridos de guerra. Basándose en su experiencia con los heridos de la Guerra de España (1936-39), y en los primeros estudios sobre el sindrome de aplastamiento (Crush
Síndrome), publicados en 1941, en los que se señalaba el fallo renal que seguía a las lesiones extensas de los miembros ${ }^{9,10}$, pudo demostrar los cambios en la circulación renal consecutivos a diferentes lesiones de los miembros, como isquemia, hemorragia e infecciones.

"Estudios sobre la circulación renal" y otros textos más breves publicados en revistas de altísimo prestigio internacional, exponen la isquemia cortical renal con preservación o aumento de la circulación medular y yuxtamedular que tiene lugar en estos estados patológicos. Los primeros estudios tuvieron su origen en la observación de los cambios de isquemia renal y oliguria que aparecen consecuencia de un traumatismo localizado en una las extremidades de un animal ${ }^{11}$. Posteriormente, en una serie de experimentos, ilustrados con extraordinarios esquemas, angiografías y preparaciones anatómicas, Trueta y sus colaboradores demuestran con numerosos métodos que en variadas situaciones patológicas, el tiempo de paso de la sangre de la arteria a la vena renal se reducía a un tercio o la mitad, en ocasiones con reducción de calibre de la arteria, y que la circulación a través de la cortical renal podía estar disminuida y en ocasiones totalmente detenida, mientras que por la medular renal aparecía una desviación del flujo sanguíneo, a través de los vasos rectos, lo que se conocería como "cortocircuito renal de Trueta” (Trueta's shunt) (Figs. 5 y 6) ${ }^{12-17}$.

Trueta creía en ese momento que el origen de la isquemia renal era un estímulo reflejo o central de los nervios vasculares, que inducía un espasmo arterial a distancia, en diversos órganos, y que este espasmo era responsable de la oliguria de los heridos en combate. Simultáneamente se describieron otras muchas causas de anoxia renal (fiebre amarilla, cólera, sangrado quirúrgico, aborto séptico), que generaron un intenso debate científico. Los experimentos de Trueta y sus hallazgos de la doble circulación renal fueron validados en otros centros europeos y norteamericanos y revalidados en 1968, cuando se volvió a destacar a la isquemia cortical del fallo renal agudo oligúrico ${ }^{4,6}$.

Aunque las hipótesis explicativas que Trueta aventuró, no fueron definitivas, sus trabajos sirvieron de referencia para otros estudios realizados simultáneamente y en los años sucesivos sobre la insuficiencia renal aguda secundaria al shock séptico, traumático y hemorrágico, la glomerulonefritis, 

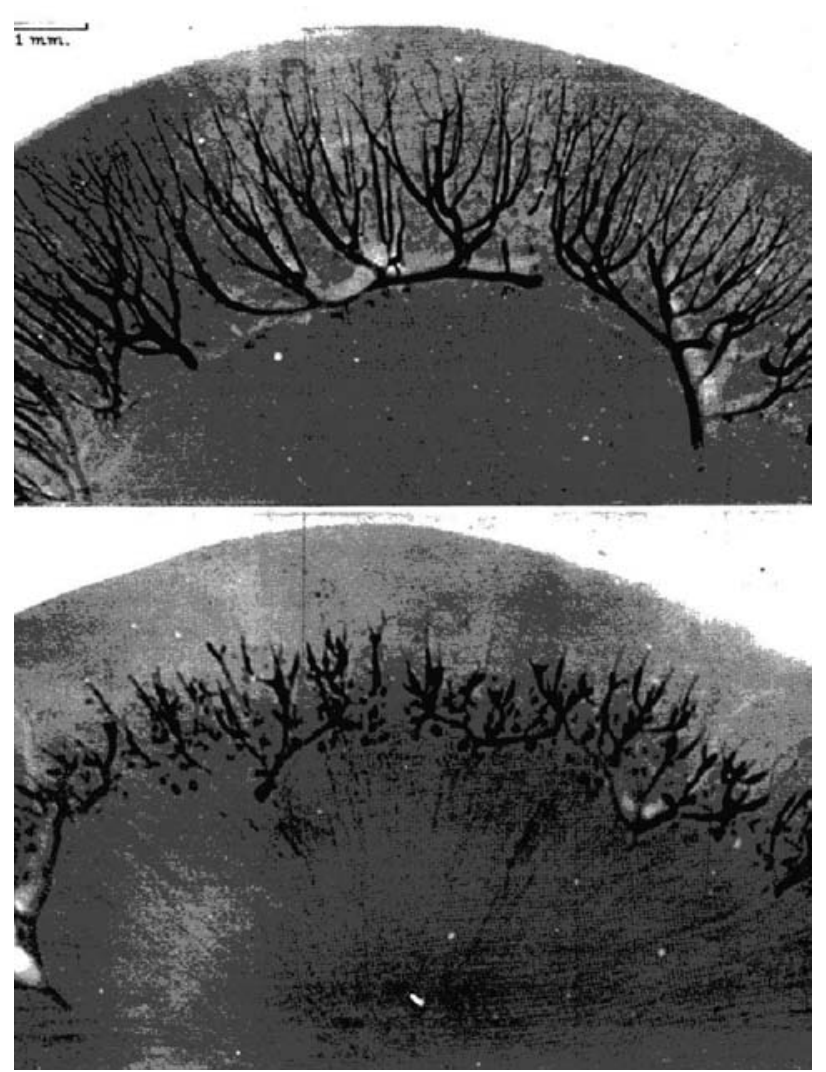

FIGURA 5. Micro-radiografias de riñón en el conejo. Arriba, una arteriografia normal, con contraste que llega a los glomérulos corticales. Abajo, en el conejo que ha sufrido un estimulo causante de isquemia renal aguda unilateral, existe un "cortocircuito renal" a través de la médula, con ausencia de circulación cortical. Tomado de Trueta et $\mathrm{al}^{8}$.

el rechazo del trasplante y la hipertensión arterial. Desde entonces, y en los últimos 50 años se ha generado un inmenso cuerpo de conocimientos sobre la enfermedad renal, que permite explicar la compleja patogénesis de la necrosis tubular aguda, que es el resultado final del daño renal generado por múltiples lesiones, no sólo de origen traumático, vascular o séptico, sino también tóxico, metabólico y de otras causas. Según el conocimiento actual, el aumento de la resistencia vascular renal y la disminución del flujo cortical son algunas de las claves iniciales de la génesis de esta lesión ${ }^{18}$.

\section{COMENTARIO}

Los trabajos de Trueta son un extraordinario ejemplo de estudios dirigidos a responder a preguntas clínicas concretas. Llama la atención el amplio campo de investigación en que se mueve, que hoy día implicaría a diversas especialidades muy ajenas a sus tareas de

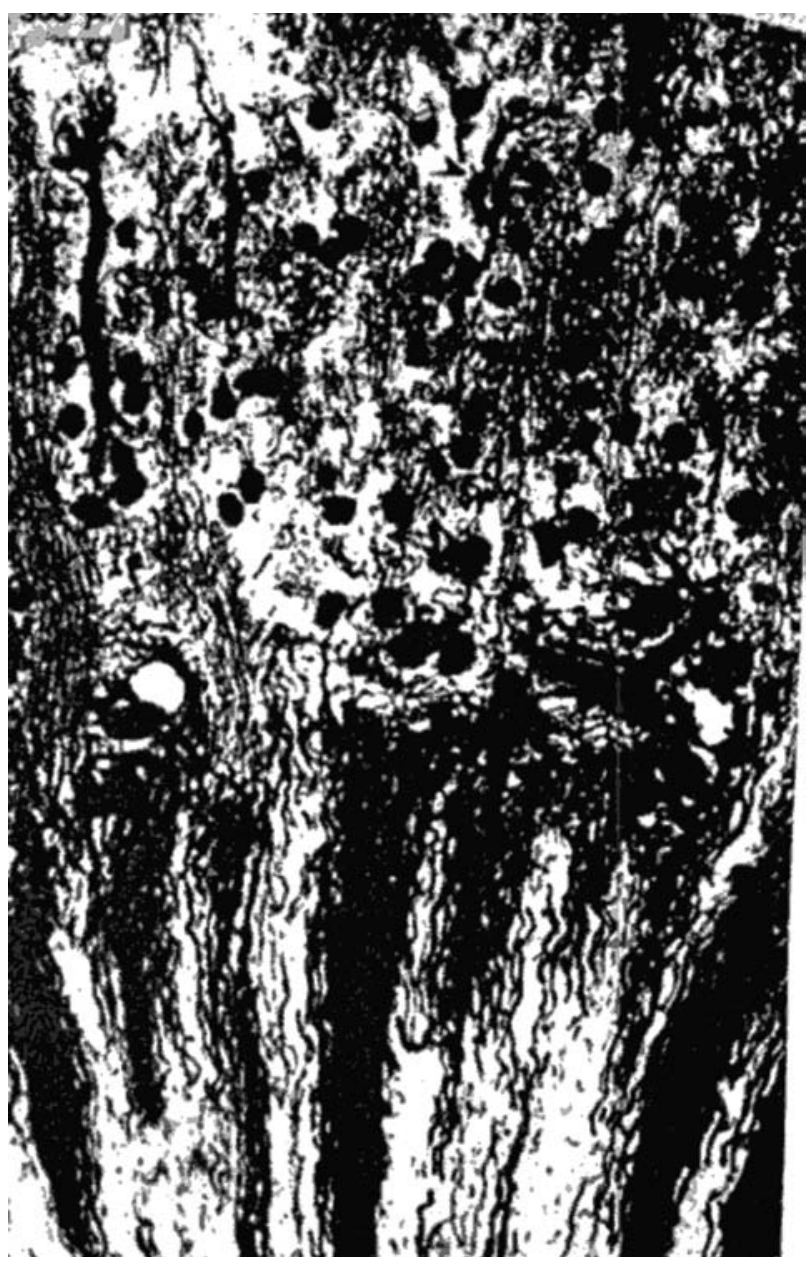

FIGURA 6. Riñón de conejo inyectado con contraste, en que se demuestran los vasa recta agrupados de forma compacta en la zona subcortical (abajo). Tomado de Trueta et $\mathrm{al}^{8}$.

cirujano ortopeda. Después de publicado este texto, el grupo de investigadores se disolvió y Trueta centró sus esfuerzos en el estudio de la organogénesis del hueso y el envejecimiento ${ }^{19}$. Por sus estudios sobre la circulación renal fue propuesto para el premio Nobel, y nombrado miembro honorario de la Societé Française d'Urologie (1951), entre otros muchos reconocimientos. Las técnicas de laboratorio que ideó para estudio de los vasos le sirvieron de experiencia para otras investigaciones sobre la circulación ósea que siguen estando vigentes en estos años ${ }^{20}$.

Trueta fue un intelectual humanista con intereses muy amplios, que dejó reflejados en sus relatos de viajes, y en textos sobre la vida de sabios clásicos catalanes y españoles, como Ramón Llull y Luís Vives, los médicos Arnau de Vilanova, Miguel Servet, y Ramón y Cajal, premio Nobel de Medicina 
en $1906^{21,22}$. Su compromiso con la libertad y con la sociedad de su tiempo, le hizo tomar parte en el primer gobierno catalán en el exilio (Consell Nacional de Catalunya), y le movió a escribir un breve texto sobre la historia de Cataluña para el público de habla inglesa ("The spirit of Catalonia”, 1946) ${ }^{23}$, posteriormente traducido a varias lenguas, que es un relato tal vez simple y maniqueo, pero sin duda emocionado, de la historia de la cultura catalana, y en especial de las contribuciones científicas y médicas hechas en los antiguos Reinos de Aragón y Cataluña en sus años de historia. Regresó a Cataluña al retirarse en el año 1966 y falleció en Santa Cristina d'Aro (Barcelona) en 1977. Su libro "Estudios sobre la circulación renal", exquisitamente ilustrado y editado, merece un lugar destacado en la historiografía médica europea de interés urológico, y le sitúa como pionero en el estudio de la insuficiencia renal aguda de origen traumático.

\section{REFERENCIAS}

1. AAVV. Josep Trueta (1897-1977). En homenatge. Fundació Barcelona, Col.legi Oficial de Metges de Barcelona. 1996.

2. Rodrigo A. Doctor Trueta. Héroe anónimo de dos guerras. Plaza y Janés, Barcelona 1977.

3. Trueta J. Sobre la projecció de l'Hospital de Santa Creu a l'estranger. En: AAVV: L'Hospital de Santa Creu i Sant Pau. L'Hospital de Barcelona. Gustau Gili, Barcelona 1986, pp 219230.

4. Cid F. Estudis sobre la circulació renal. En: Josep Trueta, esbós d'una obra médica y biológica. Tibidabo, Barcelona, 1991; pp 132-157.

5. Morlans M, Fort J, Camps J, Capdevila L, Piera L. Josep Trueta: catalan surgeon and medical researcher. Renal Failure 1996;18: 533-536.

6. Betters OS: Josep Trueta (1897-1977): Military surgeon and investigator of acute renal failure. Am J Nephrol. 1999;19:343345.
7. Trueta J, Barclay AE, Daniel PM, Franklin KJ, Prichard MML. Studies on the renal circulation. Ch C Thomas, Springfield 1947.

8. Trueta J, Barclay AE, Daniel PM, Franklin KJ, Prichard MML. Estudios sobre la circulación renal. Publicaciones Médicas José Janés, Barcelona 1949.

9. Bywaters EG, Beall D. Crush injuries with impairment of renal function. Reproducido en J Am Soc Nephrol. 1998;9:322-332.

10. Betters OS. The crush syndrome revisited (1940-1990). Nephron. 1990;55:97-103.

11. Barnes JM, Trueta J. Arterial spasm. An experimental study. Br J Surg. 1942;30:74-79.

12. Trueta J. Renal anoxia. Lancet 1945 (Sept 20):415.

13. Trueta J. Barclay AE, Daniel PM, Franklin KJ, Prichard MML. Renal pathology in the light of recent neurovascular studies. Lancet 1946 (Aug 17):237.

14. Trueta J. Barclay AE. Discussion on renal circulation. Proc Royal Soc Med. 1948;41:339-340.

15. Trueta J. Kidney. Annual Rev Physiol 1950:369-398.

16. Trueta J. The relation of hypertension to the renal circulation. Glasgow Med J. 1950;31:217-242.

17. Trueta J. The renal circulation in normal and pathological conditions. Newcastle Med J. 1953;24:131-138.

18. Neild GH. Pathogenesis of acute tubular necrosis. En: Mundy AR. The scientific basis of urology, Oxford 1999. pp 193-204 .

19. Trueta J. Studies on the developement and decay of the human frame . Saunders, Filadelfia, 1968.

20. Reichert IL, McCarthy ID, Hughes SP. The acute vascular response to intramedullary reaming. Microsphere estimation of blood flow in the intact ovine tibia. J Bone Joint Surg Br. 1995; 77:490-493.

21. Trueta J. The contribution of surgery to medical progress. Can Med Assoc J. 1948;58:427-433.

22. Trueta J. Michael Servetus and the discovery of the lesser circulation. Yale J Biol Med. 1948;21:11-15.

23. Trueta J. L'esperit de Catalunya. Edicions 62, Barcelona 2003.

Correspondencia autor: Dr. L.A. Fariña Pérez

Servicio de Urologia, Hospital Povisa

Salamanca, 5 - 36211 Vigo (Pontevedra) Tel.: 986413144

E mail autor: luisfarina@yahoo.com

Información artículo: Original - Historia de la Urología

Trabajo recibido: octubre 2007

Trabajo aceptado: noviembre 2007 